\title{
Stability of abstract dynamic equations on time scales
}

\author{
Alaa E Hamza ${ }^{1 *}$ and Karima M Oraby²
}

"Correspondence:

hamazaaeg2003@yahoo.com

1 Department of Mathematics,

Faculty of Science, Cairo University,

Cairo, Egypt

Full list of author information is

available at the end of the article

\section{算 Springer}

\begin{abstract}
In this paper, we investigate many types of stability, like uniform stability, asymptotic stability, uniform asymptotic stability, global stability, global asymptotic stability, exponential stability, uniform exponential stability, of the homogeneous first-order linear dynamic equations of the form

$$
x^{\Delta}(t)=A x(t), \quad t>t_{0}, t, t_{0} \in \mathbb{T} \quad x\left(t_{0}\right)=x_{0} \in D(A),
$$

where $A$ is the generator of a $C_{0}$-semigroup $\{T(t): t \in \mathbb{T}\} \subset L(X)$, the space of all bounded linear operators from a Banach space $X$ into itself. Here, $\mathbb{T} \subseteq \mathbb{R} \geq 0$ is a time scale which is an additive semigroup with the property that $a-b \in \mathbb{T}$ for any $a, b \in \mathbb{T}$ such that $a>b$. Finally, we give an illustrative example for a nonregressive homogeneous first-order linear dynamic equation and we investigate its stability.
\end{abstract}

\section{Introduction and preliminaries}

The history of asymptotic stability of dynamic equations on a time scale goes back to Aulbach and Hilger [3]. For a real scalar dynamic equation, stability and instability results were obtained by Gard and Hoffacker [12]. Pötzche [20] provides sufficient conditions for the uniform exponential stability in Banach spaces, as well as spectral stability conditions for time-varying systems on time scales. Doan, Kalauch, and Siegmund [10] established a necessary and sufficient condition for the existence of uniform exponential stability and characterized the uniform exponential stability of a system by the spectrum of its matrix. Properties of exponential stability of a time varying dynamic equation on a time scale have been also investigated recently by Bohner and Martynyuk [7], DaCunha [9], Du and Tien [11], Hoffacker and Tisdell [16], Martynyuk [17], and Peterson and Raffoul [19].

The theory of dynamic equations on time scales was introduced by Stefan Hilger in 1988 [14], in order to unify continuous and discrete calculus [4, 15]. A time scale $\mathbb{T}$ is a nonempty closed subset of $\mathbb{R}$. The forward jump operator $\sigma: \mathbb{T} \rightarrow \mathbb{T}$ is defined by $\sigma(t)=$ $\inf \{s \in \mathbb{T}: s>t\}($ supplemented by $\inf \emptyset=\sup \mathbb{T})$ and the backward jump operator $\rho: \mathbb{T} \longrightarrow$ $\mathbb{T}$ is defined by $\rho(t)=\sup \{s \in \mathbb{T}: s<t\}$ (supplemented by $\sup \emptyset=\inf \mathbb{T}$ ). The graininess function $\mu: \mathbb{T} \rightarrow \mathbb{R}^{\geq 0}$ is given by $\mu(t)=\sigma(t)-t$. A point $t \in \mathbb{T}$ is said to be right-dense if $\sigma(t)=t$, right-scattered if $\sigma(t)>t$, left-dense if $\rho(t)=t$, left-scattered if $\rho(t)<t$, isolated if $\rho(t)<t<\sigma(t)$, and dense if $\rho(t)=t=\sigma(t)$. A time scale $\mathbb{T}$ is said to be discrete if $\mathrm{t}$ is left-scattered and right-scattered for all $t \in \mathbb{T}$, and it is called continuous if $t$ is right-dense and left-dense at the same time for all $t \in \mathbb{T}$. Suppose that $\mathbb{T}$ has the topology inherited

(c) 2012 Hamza and Oraby; licensee Springer. This is an Open Access article distributed under the terms of the Creative Commons Attribution License (http://creativecommons.org/licenses/by/2.0), which permits unrestricted use, distribution, and reproduction in any medium, provided the original work is properly cited. 
from the standard topology on $\mathbb{R}$. We define the time scale interval $[a, b]:=[a, b] \cap \mathbb{T}$. Open intervals and open neighborhoods are defined similarly. A set we need to consider is $\mathbb{T}^{k}$ which is defined as $\mathbb{T}^{k}=\mathbb{T} \backslash\{M\}$ if $\mathbb{T}$ has a left-scattered maximum $M$, and $\mathbb{T}^{k}=\mathbb{T}$ otherwise. A function $f: \mathbb{T} \rightarrow X$ is called right dense continuous, or just rd-continuous, if

(i) $f$ is continuous at every right-dense point $t \in \mathbb{T}$;

(ii) $\lim _{s \rightarrow t^{-}} f(s)$ exists (finite) for every left-dense point $t \in \mathbb{T}$.

The set of rd-continuous functions $f: \mathbb{T} \rightarrow X$ will be denoted by $C_{\mathrm{rd}}=C_{\mathrm{rd}}(\mathbb{T})=C_{\mathrm{rd}}(\mathbb{T}, X)$.

A function $f: \mathbb{T} \rightarrow X$ is called delta differentiable (or simply differentiable) at $t \in \mathbb{T}^{k}$ provided there exists an $\alpha$ such that for every $\epsilon>0$ there is a neighborhood $U$ of $t$ with

$$
\|f(\sigma(t))-f(s)-\alpha(\sigma(t)-s)\| \leq \epsilon|\sigma(t)-s| \quad \text { for all } s \in U \text {. }
$$

In this case, we denote the $\alpha$ by $f^{\Delta}(t)$; and if $f$ is differentiable for every $t \in \mathbb{T}^{k}$, then $f$ is said to be differentiable on $\mathbb{T}$. If $f$ is differentiable at $t \in \mathbb{T}^{k}$, then it is easy to see that

$$
f^{\Delta}(t)= \begin{cases}\frac{f(\sigma(t))-f(t)}{\mu(t)}, & \text { if } \mu(t)>0 \\ \lim _{s \rightarrow t} \frac{f(t)-f(s)}{t-s}, & \text { if } \mu(t)=0 .\end{cases}
$$

A function $F: \mathbb{T} \rightarrow X$ is called an antiderivative of $f: \mathbb{T} \rightarrow X$ if $F^{\Delta}(t)=f(t), t \in \mathbb{T}^{k}$. The Cauchy integral is defined by

$$
\int_{s}^{t} f(\tau) \Delta \tau=F(t)-F(s), \quad s, t \in \mathbb{T},
$$

where $F$ is an antiderivative of $f$. Every rd-continuous function $f: \mathbb{T} \rightarrow X$ has an antiderivative and $F(t)=\int_{s}^{t} f(\tau) \Delta \tau$ is an antiderivative of $f$, i.e., $F^{\Delta}(t)=f(t), t \in \mathbb{T}^{k}$. Equations which include $\Delta$-derivatives are called dynamic equations. We refer the reader to the very interesting monographs of Bohner and Peterson $[5,6]$.

Definition 1.1 A mapping $A: \mathbb{T} \rightarrow L(X)$ is called regressive if $I+\mu(t) A(t)$ is invertible for every $t \in \mathbb{T}$, and we say that

$$
x^{\Delta}(t)=A(t) x(t), \quad t \in \mathbb{T}
$$

is regressive if $A$ is regressive. We say that a real valued function $p(t)$ on $\mathbb{T}$ is regressive (resp. positively regressive) if $1+\mu(t) p(t) \neq 0$ (resp. $1+\mu(t) p(t)>0), t \in \mathbb{T}$. The family of all regressive functions (resp. positively regressive functions) is denoted by $\mathcal{R}$ (resp. $\mathcal{R}^{+}$).

It is well known that if $A \in B C_{\mathrm{rd}} \mathcal{R}(\mathbb{T}, L(X))$, the space of all right dense continuous and regressive bounded functions from $\mathbb{T}$ to $L(X)$, then the initial value problem (IVP)

$$
x^{\Delta}(t)=A(t) x(t), \quad t \in \mathbb{T}, \quad x(s)=x_{s} \in X
$$

has the unique solution

$$
x(t)=e_{A}(t, s) x_{s} .
$$


Here, $e_{A}(t, s)$ is the exponential operator function. For more details, see [2]. When $X=\mathbb{R}$ and $A(t)=p(t)$ is a real valued function, Eq. (1.1) yields

$$
x^{\Delta}(t)=p(t) x(t), \quad t \in \mathbb{T}, \quad x(s)=1,
$$

whose solution has the closed form

$$
x(t)=e_{p}(t, s)=\exp \int_{s}^{t} \xi_{\mu(\tau)}(p(\tau)) \Delta \tau
$$

where

$$
\xi_{\mu}(z)= \begin{cases}\frac{1}{\mu} \log (1+\mu z), & \text { if } \mu>0 ; \\ z, & \text { if } \mu=0,\end{cases}
$$

and $\log z=\log |z|+i \arg z,-\pi<\arg z \leq \pi$ is the principal logarithm function. It is evident that when $p(\tau) \geq 0, \tau \in \mathbb{T}$, then

$$
\begin{aligned}
\xi_{\mu(\tau)}(p(\tau)) & = \begin{cases}\frac{1}{\mu(\tau)} \log (1+\mu(\tau) p(\tau)), & \text { if } \mu(\tau)>0 ; \\
p(\tau), & \text { if } \mu(\tau)=0\end{cases} \\
& =\lim _{u \rightarrow \mu(\tau)^{+}} \frac{\log (1+u p(\tau))}{u} .
\end{aligned}
$$

It can be seen that for $\lambda>0$ with $-\lambda \in \mathcal{R}^{+}$, the following claim is true

$$
e_{-\lambda}(t, \tau) \leq e^{-\lambda(t-\tau)}, \quad t \geq \tau, t, \tau \in \mathbb{T} .
$$

Indeed, by taking $p(\tau)=-\lambda$ in Eqs. (1.5) and (1.6), we have

$$
\begin{aligned}
\xi_{\mu(s)}(-\lambda) & =\left\{\begin{array}{ll}
\frac{1}{\mu(s)} \ln (1-\lambda \mu(s)), & \text { for } \mu(s)>0 ; \\
-\lambda, & \text { for } \mu(s)=0,
\end{array} \quad s \in \mathbb{T}\right. \\
& =\lim _{u \rightarrow \mu(s)^{+}} \frac{\log (1-\lambda u)}{u} \\
& \leq-\lambda .
\end{aligned}
$$

This implies that the claim is true.

In the sequel, we denote by $\mathbb{T} \subseteq \mathbb{R}^{\geq 0}$ for a time scale which is an additive semigroup with the property that $a-b \in \mathbb{T}$ for any $a, b \in \mathbb{T}$ such that $a>b$. In this case, $\mathbb{T}$ is called a semigroup time scale. We assume $X$ is a Banach space. Finally, we assume that $T=\{T(t)$ : $t \in \mathbb{T}\} \subset L(X)$ is a $C_{0}$-semigroup on $\mathbb{T}$, that is, it satisfies

(i) $T(t+s)=T(t) T(s)$ for every $t, s \in \mathbb{T}$ (the semigroup property).

(ii) $T(0)=I$ ( $I$ is the identity operator on $X$ ).

(iii) $\lim _{t \rightarrow 0^{+}} T(t) x=x($ i.e., $T(\cdot) x: \mathbb{T} \longrightarrow X$ is continuous at 0 ) for each $x \in X$. 
If in addition $\lim _{t \rightarrow 0^{+}}\|T(t)-I\|=0$, then $T$ is called a uniformly continuous semigroup. A linear operator $A$ is called the generator [1] of a $C_{0}$-semigroup $T$ if

$$
A x=\lim _{s \rightarrow 0^{+}} \frac{T(\mu(t)) x-T(s) x}{\mu(t)-s}, \quad x \in D(A),
$$

where the domain $D(A)$ of $A$ is the set of all $x \in X$ for which the above limit exists uniformly in $t$. Clearly, when $\mathbb{T}=\mathbb{R}^{\geq 0}$, the concept of the generator defined by relation (1.10) coincides with the classical definition by Hille. See [13].

In Section 2 of this paper we present some results from [1] that we need in our study. One of them is that an abstract Cauchy problem

$$
x^{\Delta}(t)=A x(t), \quad t>\tau, t, \tau \in \mathbb{T}, \quad x(\tau)=x_{\tau} \in D(A)
$$

has the unique solution

$$
x(t)=T(t-\tau) x_{\tau}, \quad t \in \mathbb{T}, t \geq \tau
$$

when $A$ is the generator of the $C_{0}$-semigroup $T$. When $\mathbb{T}=\mathbb{R}^{\geq 0}$, we get the classical existence and uniqueness theorem of the abstract Cauchy problem (1.11); see [21]. The other results include some properties of $T$ and its generator $A$, which we use in the subsequent sections. The solution $x(t)=x\left(t, \tau, x_{\tau}\right)$ is a function of the variables $t, \tau$ and the initial value $x_{\tau}$. Generally, we consider $\tau$ and $x_{\tau}$ as parameters. Therefore, when we investigate the asymptotic behavior of $x\left(t, \tau, x_{\tau}\right)$ with respect to $\mathbb{T}$, we must investigate whether or not the asymptotic behavior uniformly depends on $\tau$ or $x_{\tau}$. Accordingly, there are many types of stability which we give in Section 3.

S. K. Choi, D. M. Im, and N. Koo in [8, Theorem 3.5] proved that the stability of the time variant abstract Cauchy problem

$$
x^{\Delta}(t)=A(t) x(t), \quad x\left(t_{0}\right)=x_{0}, \quad t_{0} \in \mathbb{T},
$$

where $A \in C_{\mathrm{rd}} \mathcal{R}\left(\mathbb{T}, M_{n}(\mathbb{R})\right), n \in \mathbb{N}$ and $M_{n}(\mathbb{R})$ is the family of all $n \times n$ real matrices is equivalent to the boundedness of all its solutions. DaCunha in [9] defined the concepts of uniform stability and uniform exponential stability. These two concepts involve the boundedness of the solutions of the regressive time varying linear dynamic Eq. (1.12). He established a characterization of uniform stability and uniform exponential stability in terms of the transition matrix for system (1.12). Also, he illustrated the relationship between the uniform asymptotic stability and the uniform exponential stability.

In Section 4, we extend these results for the case where $A$ is the generator of $T$ and we prove that the concepts of stability and uniform stability are same.

Sections 5 and 6 are devoted to establishing characterizations for many other types of stability, like asymptotic stability, uniform asymptotic stability, global asymptotic stability, exponential stability, and uniform exponential stability for the abstract Cauchy problem (1.11). 
We end this paper with a new illustrative example including non-regressive dynamic equation and we investigate its stability.

\section{The existence and uniqueness of solutions of dynamic equations}

Our aim in this section is to prove that the first order initial value problem

$$
x^{\Delta}(t)=A x(t), \quad t \in \mathbb{T}, \quad x(0)=x \in D(A)
$$

has the unique solution

$$
x(t)=T(t) x, \quad t \in \mathbb{T},
$$

when $A$ is the generator of a $C_{0}$-semigroup $T=\{T(t): t \in \mathbb{T}\}$.

At first, we establish some properties of $T$ and its generator $A$ which we use to arrive at our aim.

Theorem 2.1 For $x \in X$, the following statements are true:

1. For $t \in \mathbb{T}$,

$$
\begin{aligned}
\lim _{s \rightarrow t} \frac{1}{s-\sigma(t)} \int_{\sigma(t)}^{s} T(\tau) x \Delta \tau & =\lim _{h \rightarrow 0} \frac{1}{h-\mu(t)} \int_{\mu(t)+t}^{h+t} T(\tau) x \Delta \tau \\
& =T(t) x
\end{aligned}
$$

and

$$
\lim _{h \rightarrow 0} \frac{1}{h-\mu(t)} \int_{\mu(t)}^{h} T(\tau) x \Delta \tau=x .
$$

2. For $t \in \mathbb{T}$,

$$
\int_{0}^{t} T(\tau) x \Delta \tau \in D(A)
$$

and

$$
A\left(\int_{0}^{t} T(\tau) x \Delta \tau\right)=T(t) x-x
$$

Proof 1 . Set $f(t)=\int_{0}^{t} T(\tau) x \Delta \tau$. Then

$$
\begin{aligned}
\lim _{s \rightarrow t} \frac{1}{s-\sigma(t)} \int_{\sigma(t)}^{s} T(\tau) x \Delta \tau & =\lim _{s \rightarrow t} \frac{1}{s-\sigma(t)}\left[\int_{0}^{s} T(\tau) x \Delta \tau-\int_{0}^{\sigma(t)} T(\tau) x \Delta \tau\right] \\
& =\lim _{s \rightarrow t} \frac{f(s)-f(\sigma(t)}{s-\sigma(t)} \\
& =f^{\Delta}(t) \\
& =T(t) x .
\end{aligned}
$$


Also, we have

$$
\begin{aligned}
\lim _{h \rightarrow 0} \frac{1}{h-\mu(t)} \int_{\mu(t)+t}^{h+t} T(s) x \Delta s & =\lim _{h \rightarrow 0} \frac{f(h+t)-f(\mu(t)+t)}{h-\mu(t)} \\
& =f^{\Delta}(t) \\
& =T(t) x,
\end{aligned}
$$

and

$$
\begin{aligned}
\lim _{h \rightarrow 0} \frac{1}{h-\mu(t)} \int_{\mu(t)}^{h} T(s) x \Delta s & =\lim _{u \rightarrow t^{+}} \frac{1}{u-\sigma(t)} \int_{\sigma(t)-t}^{u-t} T(s) x \Delta s \\
& =\lim _{u \rightarrow t^{+}} \frac{1}{u-\sigma(t)} \int_{\sigma(t)}^{u} T(s-t) x \Delta s \\
& =\phi^{\Delta}(t) \\
& =x,
\end{aligned}
$$

where $\phi(u)=\int_{t}^{u} T(s-t) x \Delta s$.

2. Let $h>0$ be a number in $\mathbb{T}$. We have

$$
\begin{aligned}
A \int_{0}^{t} T(s) x \Delta s & =\lim _{h \rightarrow 0^{+}} \frac{1}{\mu(t)-h}\left[\int_{0}^{t} T(\mu(t)+s) x \Delta s-\int_{0}^{t} T(h+s) x \Delta s\right] \\
& =\lim _{h \rightarrow 0^{+}} \frac{1}{\mu(t)-h}\left[\int_{t+h}^{\mu(t)+t} T(s) x \Delta s+\int_{\mu(t)}^{h} T(s) x \Delta s\right] \\
& =T(t) x-x \quad \text { by Eqs. (2.3), (2.4). }
\end{aligned}
$$

Theorem 2.2 For $x \in D(A)$, the following statements are true:

1. For $t \in \mathbb{T}, x(t)=T(t) x \in D(A)$ and

$$
x^{\Delta}(t)=A T(t) x=T(t) A x .
$$

2. For $t, s \in \mathbb{T}$, we have

$$
\begin{aligned}
T(t) x-T(s) x & =\int_{s}^{t} T(\tau) A x \Delta \tau \\
& =\int_{s}^{t} A T(\tau) x \Delta \tau
\end{aligned}
$$

and

$$
A \int_{0}^{t} T(\tau) x \Delta \tau=\int_{0}^{t} T(\tau) A x \Delta \tau .
$$

Proof 1. Let $x \in D(A)$. It is evident that $T(t) x \in D(A), t \in \mathbb{T}$.

Now, we show that $x(t)=T(t) x$ solves the initial value problem

$$
x^{\Delta}(t)=A x(t), \quad x(0)=x .
$$


We have either $\mu(t)=0$ or $\mu(t)>0$. The case $\mu(t)=0$ implies

$$
\begin{aligned}
\lim _{u \rightarrow t^{-}} \frac{x(\sigma(t))-x(u)}{\sigma(t)-u} & =\lim _{u \rightarrow t^{-}} \frac{T(t) x-T(u) x}{t-u} \\
& =\lim _{s \rightarrow 0^{+}} T(t-s) \frac{T(s) x-x}{s} \\
& =T(t) A x \\
& =A T(t) x .
\end{aligned}
$$

On the other hand,

$$
\begin{aligned}
\lim _{u \rightarrow t^{+}} \frac{x(\sigma(t))-x(u)}{\sigma(t)-u} & =\lim _{u \rightarrow t^{+}} \frac{T(t) x-T(u) x}{t-u} \\
& =\lim _{s \rightarrow 0^{+}} T(t) \frac{x-T(s) x}{-s} \\
& =T(t) A x \\
& =A T(t) x .
\end{aligned}
$$

When $\mu(t)>0$, we obtain

$$
\begin{aligned}
\lim _{u \rightarrow t} \frac{T(\sigma(t)) x-T(u) x}{\sigma(t)-u} & =\frac{T(\mu(t)+t) x-T(t) x}{\mu(t)} \\
& =T(t) \frac{T(\mu(t)) x-x}{\mu(t)} \\
& =T(t) A x \\
& =A T(t) x .
\end{aligned}
$$

2. Relations (2.7) and (2.8) can be obtained by integrating both sides of Eq. (2.6) from $s$ to $t$. Relation (2.9) follows from Eqs. (2.5) and (2.7).

Corollary 2.3 If $A$ is the generator of a $C_{0}$-semigroup $T$ on $\mathbb{T}$, then $D(A)$ is dense in $X$ and $A$ is a closed linear operator.

Proof For every $x \in X$ and fixed $t \in \mathbb{T}$, set

$$
x_{h}=\frac{1}{h-\mu(t)} \int_{\mu(t)}^{h} T(s) x \Delta s, \quad h \in \mathbb{T} .
$$

Theorem 2.1 implies that

$$
\int_{\mu(t)}^{h} T(s) x \Delta s=\int_{0}^{h} T(s) x \Delta s-\int_{0}^{\mu(t)} T(s) x \Delta s \in D(A) .
$$

By the same theorem, $x_{h} \longrightarrow x$ as $h \longrightarrow 0$. So $\overline{D(A)}$, the closure of $D(A)$, is equal to $X$. The linearity of $A$ is evident. 
To prove its closeness, let $x_{n} \in D(A), x_{n} \longrightarrow x$ and $A x_{n} \longrightarrow y$ as $n \longrightarrow \infty$. In view of equality (2.7), we obtain

$$
T(h) x_{n}-T(\mu(t)) x_{n}=\int_{\mu(t)}^{h} T(s) A x_{n} \Delta s .
$$

The integrand on the right-hand side of (2.10) converges to $T(s) y$ uniformly on bounded intervals. Consequently, letting $n \longrightarrow \infty$ in (2.10), we get

$$
T(h) x-T(\mu(t)) x=\int_{\mu(t)}^{h} T(s) y \Delta s .
$$

Dividing Eq. (2.11) by $h-\mu(t), h>0$ and letting $h \longrightarrow 0$, we see, using identity (2.4), that $x \in D(A)$ and $A x=y$.

Theorem 2.4 Equation (2.1) has the unique solution

$$
x(t)=T(t) x, \quad t \in \mathbb{T} .
$$

Proof The existence of the solution $x(t)=T(t) x$ follows by Theorem 2.2. To prove the uniqueness, assume that $V(t)$ is another solution. Consider the function

$$
G(s):=H_{t}(s) V(s), \quad s \in[0, t], s, t \in \mathbb{T},
$$

where $H_{t}(s)=T(t-s)$. We have

$$
\begin{aligned}
G^{\Delta}(s) & =H_{t}(\sigma(s)) V^{\Delta}(s)+H_{t}^{\Delta}(s) V(s) \\
& =T(t-\sigma(s)) A V(s)+H_{t}^{\Delta}(s) V(s) .
\end{aligned}
$$

On the other hand, we have

$$
\begin{aligned}
H_{t}^{\Delta}(s) x & =\lim _{u \rightarrow s} \frac{T(t-\sigma(s))-T(t-u)}{\sigma(s)-u} x \\
& =\lim _{r \rightarrow 0} T(t-\sigma(s)) \frac{I-T(\mu(s)-r)}{\mu(s)-r} x \\
& =-T(t-\sigma(s)) A x,
\end{aligned}
$$

from which we obtain that $G^{\Delta}(s)=0$ on $[0, t[$. Then $G(t)=G(0)$, i.e. $V(t)=T(t) V(0)=$ $T(t) x$.

\section{Types of stability}

In this section, the definitions of the various types of stability for dynamic equations of the form

$$
x^{\Delta}(t)=F(t, x), \quad x\left(t_{0}\right)=x_{0} \in X, \quad t, t_{0} \in \mathbb{T}
$$

are presented, where $F \in C_{\mathrm{rd}}(\mathbb{T} \times X, X)$ and $x^{\Delta}$ is the delta derivative of $x: \mathbb{T} \rightarrow X$ with respect to $t \in \mathbb{T}^{k}$. See $[8,18]$. 
Definition 3.1 Equation (3.1) is said to be stable if, for every $t_{0} \in \mathbb{T}$ and for every $\epsilon>0$, there exists a $\delta=\delta\left(\epsilon, t_{0}\right)>0$ such that, for any two solutions $x(t)=x\left(t, t_{0}, x_{0}\right)$ and $\bar{x}(t)=$ $x\left(t, t_{0}, \bar{x}_{0}\right)$ of Eq. (3.1), the inequality $\left\|x_{0}-\bar{x}_{0}\right\|<\delta$ implies $\|x(t)-\bar{x}(t)\|<\epsilon$, for all $t \geq t_{0}$, $t \in \mathbb{T}$.

Definition 3.2 Equation (3.1) is said to be uniformly stable if, for each $\epsilon>0$, there exists a $\delta=\delta(\epsilon)>0$ independent on any initial point $t_{0}$ such that, for any two solutions $x(t)=x\left(t, t_{0}, x_{0}\right)$ and $\bar{x}(t)=x\left(t, t_{0}, \bar{x}_{0}\right)$ of Eq. (3.1), the inequality $\left\|x_{0}-\bar{x}_{0}\right\|<\delta$ implies $\|x(t)-\bar{x}(t)\|<\epsilon$, for all $t \geq t_{0}, t \in \mathbb{T}$.

Definition 3.3 Equation (3.1) is said to be asymptotically stable if it is stable and for every $t_{0} \in \mathbb{T}$, there exists a $\delta=\delta\left(t_{0}\right)>0$ such that, the inequality $\left\|x_{0}\right\|<\delta$ implies $\lim _{t \rightarrow \infty}\|x(t)\|=0$.

Definition 3.4 Equation (3.1) is said to be uniformly asymptotically stable if it is uniformly stable and there exists a $\delta>0$ such that for every $t_{0} \in \mathbb{T}$ the inequality $\left\|x_{0}\right\|<\delta$ implies $\lim _{t \rightarrow \infty}\|x(t)\|=0, t \in \mathbb{T}$.

Definition 3.5 Equation (3.1) is said to be globally asymptotically stable if it is stable and for any solution $x(t)=x\left(t, t_{0}, x_{0}\right)$ of Eq. (3.1), we have $\lim _{t \rightarrow \infty}\|x(t)\|=0$.

Definition 3.6 Equation (3.1) is said to be exponentially stable if there exists $\alpha>0$ with $-\alpha \in \mathcal{R}^{+}$such that for every $t_{0} \in \mathbb{T}$, there is $\gamma=\gamma\left(t_{0}\right) \geq 1$ such that, for any two solutions $x(t)=x\left(t, t_{0}, x_{0}\right)$ and $\bar{x}(t)=x\left(t, t_{0}, \bar{x}_{0}\right)$ of Eq. (3.1), we have $\|x(t)-\bar{x}(t)\| \leq \gamma \| x_{0}-$ $\bar{x}_{0} \| e_{-\alpha}\left(t, t_{0}\right)$, for all $t \geq t_{0}, t \in \mathbb{T}$.

Definition 3.7 Equation (3.1) is said to be uniformly exponentially stable if there exists $\alpha>0$ with $-\alpha \in \mathcal{R}^{+}$and there is $\gamma \geq 1$ independent on any initial point $t_{0}$ such that, for any two solutions $x(t)=x\left(t, t_{0}, x_{0}\right)$ and $\bar{x}(t)=x\left(t, t_{0}, \bar{x}_{0}\right)$ of Eq. (3.1), we have $\|x(t)-\bar{x}(t)\| \leq$ $\gamma\left\|x_{0}-\bar{x}_{0}\right\| e_{-\alpha}\left(t, t_{0}\right)$, for all $t \geq t_{0}, t \in \mathbb{T}$.

\section{Characterization of stability and uniformly stability}

In this section, we obtain some results concerning characterizations of stability and uniform stability of linear dynamic equations of the form

$$
C P(0): \quad x^{\Delta}(t)=A x(t), \quad x\left(t_{0}\right)=x_{0} \in D(A), \quad t \geq t_{0}, t, t_{0} \in \mathbb{T},
$$

where $A$ is the generator of $T$. The initial value problem $C P(0)$ has the unique solution

$$
x(t)=T\left(t-t_{0}\right) x_{0}
$$

In the following two lemmas, by linearity of $C P(0)$, we get an equivalent definition of stability and uniform stability of $C P(0)$.

Lemma 4.1 The following statements are equivalent:

(i) $\mathrm{CP}(0)$ is stable; 
(ii) For every $t_{0} \in \mathbb{T}$ and for every $\epsilon>0$, there exists $\delta=\delta\left(\epsilon, t_{0}\right)$ such that for any solution $x(t)=x\left(t, t_{0}, x_{0}\right)$ of $C P(0)$, we have

$$
\left\|x_{0}\right\|<\delta \quad \Longrightarrow \quad\|x(t)\|<\epsilon .
$$

Lemma 4.2 The following statements are equivalent:

(i) $C P(0)$ is uniformly stable;

(ii) For every $\epsilon>0$ there exists $\delta=\delta(\epsilon)$ such that for any solution $x(t)=x\left(t, t_{0}, x_{0}\right)$ of $C P(0)$, we have

$$
\left\|x_{0}\right\|<\delta \quad \Longrightarrow \quad\|x(t)\|<\epsilon \text {. }
$$

S. K. Choi, D. M. Im, and N. Koo in [8, Theorem 3.5] proved that the stability of (1.12) is equivalent to the boundedness of all its solutions when $A \in C_{\mathrm{rd}} \mathcal{R}\left(\mathbb{T}, M_{n}(\mathbb{R})\right), n \in \mathbb{N}$ where $M_{n}(\mathbb{R})$ is the family of all $n \times n$ real matrices. Also, DaCunha in [9] proved that the uniform stability of (1.12) is equivalent to the uniform boundedness of all its solutions with respect to the initial point $t_{0}$, when $A \in C_{\mathrm{rd}} \mathcal{R}\left(\mathbb{T}, M_{n}(\mathbb{R})\right)$.

In the following theorem, we extend these results for the case where $A$ is the generator of a $C_{0}$-semigroup $T$ and we prove that the concepts of stability and uniform stability are the same.

Theorem 4.3 The following statements are equivalent:

(i) $C P(0)$ is stable.

(ii) $\{T(t): t \in \mathbb{T}\}$ is bounded.

(iii) $C P(0)$ is uniformly stable.

Proof (i) $\Longrightarrow$ (ii) Assume $C P(0)$ is stable. Let $t_{0} \in \mathbb{T}$. Fix $\epsilon=1$. There exists $\delta>0$ such that for any solution $x(t)=T\left(t-t_{0}\right) x_{0}$, where $x_{0} \in D(A)$, we have

$$
\left\|x_{0}\right\|<\delta \quad \Longrightarrow \quad\left\|T\left(t-t_{0}\right) x_{0}\right\|<1, \quad \forall t \geq t_{0}, t \in \mathbb{T} .
$$

Let $0 \neq y_{0} \in D(A)$. Take $x_{0}=\frac{\delta y_{0}}{2\left\|y_{0}\right\|}$. Since $\left\|x_{0}\right\|<\delta$, then

$$
\left\|T\left(t-t_{0}\right) \frac{\delta y_{0}}{2\left\|y_{0}\right\|}\right\|<1
$$

i.e.

$$
\left\|T\left(t-t_{0}\right) y_{0}\right\|<\frac{2}{\delta}\left\|y_{0}\right\|, \quad \forall y_{0} \in D(A) \forall t \geq t_{0}, t \in \mathbb{T} .
$$

The density of $D(A)$ in $X$, by Corollary 2.3, implies that

$$
\left\|T\left(t-t_{0}\right) x\right\|<\frac{2}{\delta}\|x\|, \quad \forall x \in X \forall t \geq t_{0}, t \in \mathbb{T} .
$$

Thus, for every $x \in X,\left\{\left\|T\left(t-t_{0}\right) x\right\|: t \in \mathbb{T}, t \geq t_{0}\right\}$ is bounded. By the uniform boundedness theorem [22], $\{\|T(t)\|: t \in \mathbb{T}\}$ is bounded.

(ii) $\Longrightarrow$ (iii) Assume that there is $M>0$ such that $\|T(t)\| \leq M, t \in \mathbb{T}$. Clearly, condition (ii) of Lemma 4.2 holds, because for $\epsilon>0$, choose $\delta=\epsilon / M$. 


\section{A characterization of global asymptotic stability}

In the following result, we establish necessary and sufficient conditions for $C P(0)$ to be globally asymptotically stable.

Theorem 5.1 The following statement are equivalent:

(i) $C P(0)$ is asymptotically stable;

(ii) $\lim _{t \rightarrow \infty}\|T(t) x\|=0$, for every $x \in X$;

(iii) $C P(0)$ is globally asymptotically stable;

(iv) $C P(0)$ is uniformly asymptotically stable.

Proof (i) $\Longrightarrow$ (ii) Suppose that $C P(0)$ is asymptotically stable. Let $t_{0} \in \mathbb{T}$. There exists $\gamma=$ $\gamma\left(t_{0}\right)>0$ such that any solution $x(t)=x\left(t, t_{0}, x_{0}\right)$ of $C P(0)$ with initial value $x_{0} \in D(A)$, vanishes at $\infty$ whenever $\left\|x_{0}\right\|<\gamma$. Fix $0 \neq x \in D(A)$. Then

$$
\lim _{t \rightarrow \infty}\left\|T\left(t-t_{0}\right) \gamma x /(2\|x\|)\right\|=0 .
$$

Hence,

$$
\lim _{t \rightarrow \infty}\left\|T\left(t-t_{0}\right) x\right\|=0, \quad x \in D(A) .
$$

Consequently, we obtain

$$
\lim _{t \rightarrow \infty}\|T(t) x\|=0, \quad x \in D(A) .
$$

By the boundedness of $\{T(t): t \in \mathbb{T}\}$ and the density of $D(A)$ in $X$, we deduce that

$$
\lim _{t \rightarrow \infty}\|T(t) x\|=0, \quad x \in X
$$

(ii) $\Longrightarrow$ (iii) Condition (ii) implies that $\{\|T(t) x\|: t \in \mathbb{T}\}$ is bounded for every $x \in X$. The uniform boundedness theorem insures the boundedness of $\{\|T(t)\|: t \in \mathbb{T}\}$. Consequently, $C P(0)$ is stable, and by our assumption, $C P(0)$ is globally asymptotically stable.

(iii) $\Longrightarrow$ (iv) Condition (iii) implies that $\{\|T(t) x\|: t \in \mathbb{T}\}$ is bounded for every $x \in X$. Again the uniform boundedness theorem guarantees the boundedness of $\{\|T(t)\|: t \in \mathbb{T}\}$. Consequently, $C P(0)$ is uniformly stable by Theorem 4.3 , and by our assumption, $C P(0)$ is uniformly asymptotically stable.

\section{A characterization of exponential stability and uniform exponential stability}

We need the following lemmas to establish a characterization of the exponential stability of $C P(0)$. Their proofs are straightforward and will be omitted.

Lemma 6.1 $C P(0)$ is exponentially stable if and only if there exists $\alpha>0$ with $-\alpha \in \mathcal{R}^{+}$such that for any $t_{0} \in \mathbb{T}$, there exists $\gamma=\gamma\left(t_{0}\right) \geq 1$ such that for any solution $x(t)=x\left(t, t_{0}, x_{0}\right)$ of $C P(0)$ with initial value $x_{0} \in D(A)$ we have

$$
\|x(t)\| \leq \gamma\left\|x_{0}\right\| e_{-\alpha}\left(t, t_{0}\right), \quad t \geq t_{0}, t \in \mathbb{T} .
$$


Lemma 6.2 $C P(0)$ is uniformly exponentially stable if and only if there exists $\alpha>0$ with $-\alpha \in \mathcal{R}^{+}$and there exists $\gamma \geq 1$ such that for any $t_{0} \in \mathbb{T}$, and any solution $x(t)=x\left(t, t_{0}, x_{0}\right)$ of $C P(0)$ with initial value $x_{0} \in D(A)$ we have

$$
\|x(t)\| \leq \gamma\left\|x_{0}\right\| e_{-\alpha}\left(t, t_{0}\right), \quad t \geq t_{0}, t \in \mathbb{T} .
$$

In the following two theorems, we extend the results of DaCunha [9, Theorem 2.2] when $A \in C_{\mathrm{rd}} \mathcal{R}\left(\mathbb{T}, M_{n}(\mathbb{R})\right)$ to the case where $A$ is the generator of $T$.

Theorem 6.3 The following statements are equivalent:

(i) $C P(0)$ is exponentially stable;

(ii) There exists $\alpha>0$ with $-\alpha \in \mathcal{R}^{+}$such that for any $t_{0} \in \mathbb{T}$, there exists $\gamma=\gamma\left(t_{0}\right) \geq 1$ such that

$$
\|T(t)\| \leq \gamma e_{-\alpha}\left(t+t_{0}, t_{0}\right), \quad t \in \mathbb{T} .
$$

Proof (i) $\Longrightarrow$ (ii) Let $C P(0)$ be exponentially stable. Then there is $\alpha>0$ with $-\alpha \in \mathcal{R}^{+}$such that for any $t_{0} \in \mathbb{T}$, there exists $\gamma=\gamma\left(t_{0}\right) \geq 1$ such that for any solution $x(t)=T\left(t-t_{0}\right) x_{0}$ of $C P(0)$ with initial value $x_{0} \in D(A)$, we have

$$
\left\|T\left(t-t_{0}\right) x_{0}\right\| \leq \gamma\left\|x_{0}\right\| e_{-\alpha}\left(t, t_{0}\right), \quad t \geq t_{0}, t \in \mathbb{T} .
$$

Fix $t_{0} \in \mathbb{T}$, and let $0 \neq x \in D(A)$. Then

$$
\left\|T\left(t-t_{0}\right) x\right\| \leq \gamma\|x\| e_{-\alpha}\left(t, t_{0}\right), \quad t \geq t_{0}, t \in \mathbb{T} .
$$

Using $D(A)$ is dense in $X$ and Corollary 2.3, we obtain

$$
\left\|T\left(t-t_{0}\right) x\right\| \leq \gamma\|x\| e_{-\alpha}\left(t, t_{0}\right), \quad x \in X, t \geq t_{0}, t \in \mathbb{T} .
$$

This implies that

$$
\|T(t)\| \leq \gamma e_{-\alpha}\left(t+t_{0}, t_{0}\right), \quad t \in \mathbb{T} .
$$

(ii) $\Longrightarrow$ (i) Assume there exists $\alpha>0$ with $-\alpha \in \mathcal{R}^{+}$such that for every $t_{0} \in \mathbb{T}$, there exists $\gamma=\gamma\left(t_{0}\right) \geq 1$ such that

$$
\|T(t)\| \leq \gamma e_{-\alpha}\left(t+t_{0}, t_{0}\right), \quad t \in \mathbb{T} .
$$

Let $x\left(t, t_{0}, x_{0}\right)=T\left(t-t_{0}\right) x_{0}$ be any solution of $C P(0)$ with initial value $x_{0}$. Then

$$
\begin{aligned}
\|x(t)\| & =\left\|T\left(t-t_{0}\right) x_{0}\right\| \\
& \leq\left\|T\left(t-t_{0}\right)\right\|\left\|x_{0}\right\| \\
& \leq \gamma e_{-\alpha}\left(t, t_{0}\right)\left\|x_{0}\right\|, \quad t \geq t_{0}, t \in \mathbb{T} .
\end{aligned}
$$

By same way as in the proof of Theorem 6.3, we can obtain the following result. 
Theorem 6.4 The following statements are equivalent:

(i) $C P(0)$ is uniformly exponentially stable;

(ii) There exists $\alpha>0$ with $-\alpha \in \mathcal{R}^{+}$and there exists $\gamma \geq 1$ such that for any $t_{0} \in \mathbb{T}$,

$$
\|T(t)\| \leq \gamma e_{-\alpha}\left(t+t_{0}, t_{0}\right), \quad t \in \mathbb{T} .
$$

From Theorem 5.1 (Theorem 6.4), Lemma 6.1 (Lemma 6.2), and relation (1.7), we get the following result.

Corollary 6.5 If $C P(0)$ is (uniformly) exponentially stable, then $C P(0)$ is (uniformly) asymptotically stable.

\section{Example}

Choi in [8] gave an example to illustrate many types of stability. He considered the linear dynamic system

$$
x^{\Delta}(t)=A x, \quad x(0)=x_{0}, \quad t>0, t \in \mathbb{T},
$$

where $\mathbb{T} \subset \mathbb{R}^{\geq 0}$ is a time scale and $A=\left(\begin{array}{cc}0 & 0 \\ 0 & -2\end{array}\right)$ and investigated some types of stability of Eq. (7.1) when $A$ is regressive, i.e., $\mu(t) \neq \frac{1}{2}$ for all $t \in \mathbb{T}$. In this case the equation has the unique solution $x(t)=e_{A}(t, 0) x_{0}$, where $e_{A}(t, 0)$ is the matrix exponential function. It is given by

$$
e_{A}(t, 0)=\left(\begin{array}{cc}
1 & 0 \\
0 & e_{-2}(t, 0)
\end{array}\right), \quad t \in \mathbb{T}
$$

We see that the generalized exponential function $e_{-2}(t, 0)$ is given by

$$
e_{-2}(t, 0)=e^{-2 t}, \quad \text { if } t \in \mathbb{T}=\mathbb{R}^{\geq 0}
$$

and

$$
e_{-2}(t, 0)=(1-2 h)^{\frac{t}{h}}, \quad \text { if } t \in \mathbb{T}=h \mathbb{Z}^{\geq 0}, h \neq \frac{1}{2} .
$$

The following stability results [8] for (7.1) were obtained in different cases of $\mathbb{T}$.

(1) If $\mathbb{T}=\mathbb{R}^{\geq 0}$, then (7.1) is uniformly stable, exponentially stable and asymptotically stable, since $\left\|e_{A}(t, 0)\right\|=e^{-2 t} \longrightarrow 0$ as $t \longrightarrow \infty$.

(2) If $\mathbb{T}=\mathbb{Z}^{\geq 0}$, then (7.1) is uniformly stable but not asymptotically stable, since $\left\|e_{A}(t, 0)\right\|=1$.

(3) If $\mathbb{T}=h \mathbb{Z}^{\geq 0}$ with $0<h<1$ and $h \neq \frac{1}{2}$, then (7.1) is not asymptotically stable. However, $e_{-2}(t, 0)$ goes to zero as $t \longrightarrow \infty$.

(4) If $\mathbb{T}=h \mathbb{Z}^{\geq 0}$ with $h>1$, then (7.1) is not asymptotically stable.

Now we consider the time scale $\mathbb{T}=\left\{\frac{n}{2}: n \in \mathbb{Z}^{\geq 0}\right\}$ with the graininess function $\mu(t)=1 / 2$, $t \in \mathbb{T}$. So $A$ is nonregressive and the matrix exponential function $e_{A}(t, 0)$ does not exist. On the other hand, $A$ is the generator of the $C_{0}$-semigroup

$$
T(t)=\left(I+\frac{1}{2} A\right)^{2 t}, \quad t \in \mathbb{T} .
$$


Indeed, for $x \in \mathbb{R}^{2}$, we have

$$
\begin{aligned}
\lim _{s \rightarrow 0^{+}} \frac{T(\mu(t)) x-T(s) x}{\mu(t)-s} & =\lim _{s \rightarrow 0^{+}} \frac{T(1 / 2) x-T(s) x}{\frac{1}{2}-s} \\
& =2\left[\left(I+\frac{1}{2} A\right) x-x\right]
\end{aligned}
$$

$=A x$.

Then

$$
T(t)=\left(\begin{array}{ll}
1 & 0 \\
0 & 0
\end{array}\right)
$$

Consequently, $\|T(t)\|=1, t \in \mathbb{T}$ which implies that Eq. (7.1) is uniformly stable but is not asymptotically stable.

\section{Competing interests}

The authors declare that they have no competing interests.

\section{Authors' contributions}

All authors contributed equally and significantly in writing this article. All authors read and approved the final manuscript.

\section{Author details}

'Department of Mathematics, Faculty of Science, Cairo University, Cairo, Egypt. ²Department of Mathematics, Faculty of Science, Suez Canal University, Suez, Egypt.

Received: 28 February 2012 Accepted: 25 July 2012 Published: 12 August 2012

\section{References}

1. Hamza, AE, Oraby, KM: $C_{0}$-semigroups of operators on time scales. J. Math. Anal. Appl. (submitted)

2. Hamza, AE, Al-Qubaty, M: On the exponential operator functions on time scales. Adv. Dyn. Syst. Appl. 7(1), 57-80 (2012)

3. Aulbach, B, Hilger, S: Linear dynamics processes with inhomogeneous time scales. In: Nonlinear Dynamics and Quantum Dynamical Systems (Gaussing, 1990). Math. Res., vol. 59, pp. 9-20. Akademie, Berlin (1990)

4. Aulbach, B, Hilger, S: A unified approach to continuous and discrete dynamics. In: Qualitative Theory of Differential Equations (Szeged, 1988). Colloq. Math. Soc. János Bolyai, vol. 53, pp. 37-56. North-Holland, Amsterdam (1990)

5. Bohner, M, Peterson, A: Dynamic Equations on Time Scales: An Introduction with Applications. Birkhäuser, Basel (2001)

6. Bohner, M, Peterson, A: Advances in Dynamic Equations on Time Scales. Birkhäuser, Basel (2003)

7. Bohner, M, Martynyuk, AA: Elements of stability theory of A. M. Lyapunov for dynamic equations on time scales. Prikl. Mat. Meh. 43(9), 3-27 (2007). Translation in Nonlinear Dyn. Syst. Theory 7(3), 225-257 (2007)

8. Choi, SK, Im, DM, Koo, N: Stability of linear dynamic systems on time scales. Adv. Differ. Equ. 2008, Article ID 670203 (2008). doi:10.1155/2008/670203

9. DaCunha, JJ: Stability for time varying linear dynamic systems on time scales. J. Comput. Appl. Math. 176, 381-410 (2005)

10. Doan, TS, Kalauch, A, Siegmund, S: Exponential stability of linear time-invariant systems on time scales. Nonlinear Dyn. Syst. Theory 9(1), 37-50 (2009)

11. Du, NH, Tien, LH: On the exponentially stability of dynamic equations on time scales. J. Math. Anal. Appl. 331 1159-1174 (2007)

12. Gard, T, Hoffacker, J: Asymptotic behavior of natural growth on time scales. Dyn. Syst. Appl. 12(1-2), 131-148 (2003)

13. Hille, E: Functional Analysis and Semigroups. Amer. Math. Soc. Colloq. Publ., vol. 31. Am. Math. Soc., New York (1948)

14. Hilger, S: Ein Maßkettenkalkül mit Anwendung auf Zentrumsmannigfaltigkeiten. PhD thesis, Universität Würzburg (1988)

15. Hilger, S: Analysis on measure chains - a unified approach to continuous and discrete calculus. Results Math. 18 18-56 (1990)

16. Hoffacker, J, Tisdell, CC: Stability and instability for dynamics equations on time scales. Comput. Math. Appl. 49 1327-1334 (2005)

17. Martynyuk, AA: On exponential stability of a dynamical system on a time scale. Dokl. Akad. Nauk SSSR 421, 312-317 (2008)

18. Al-Qubaty, M: On theory of dynamics equations on time scales. PhD thesis, Ain Shams University (2010)

19. Peterson, A, Raffoul, RF: Exponential stability of dynamic equations on time scales. Adv. Differ. Equ. 2005, 133-144 (2005) 
20. Pötzche, C, Siegmund, S, Wirth, F: A spectral characterization of exponential stability for linear time-invariant systems on time scales. Discrete Contin. Dyn. Syst. 9, 1223-1241 (2003)

21. Pazy, A: Semigroups of Linear Operators and Applications to Partial Differential Equations (1983)

22. Yosida, K: Functional Analysis, 6th edn. Springer, Berlin (1980)

doi:10.1186/1687-1847-2012-143

Cite this article as: Hamza and Oraby: Stability of abstract dynamic equations on time scales. Advances in Difference Equations 2012 2012:143.

Submit your manuscript to a SpringerOpen ${ }^{\circ}$ journal and benefit from:

- Convenient online submission

- Rigorous peer review

- Immediate publication on acceptance

Open access: articles freely available online

- High visibility within the field

- Retaining the copyright to your article

Submit your next manuscript at $>$ springeropen.com 